\title{
Estimation of masticatory forces for patient-specific analysis of the human mandible
}

\author{
M. Koseki ${ }^{1}$, N. Inou ${ }^{1} \&$ K. Maki ${ }^{2}$ \\ ${ }^{1}$ Department of Mechanical and Control Engineering, \\ Tokyo Institute of Technology, Japan \\ ${ }^{2}$ Department of Orthodontics, Showa University, Japan
}

\begin{abstract}
This paper proposes an estimation method of the masticatory forces using an objective function composed of three criteria: efficiency of muscular activities, moment balance between muscular and biting forces, and reaction forces at condyles. The method is applied to a patient whose jaw has a severe deformity, and the influence of the criteria is examined. The muscular forces of the numerical result with the consideration of all three criteria well agree to the analysis of EMG signals. Individual stress analysis under the estimated mechanical condition is also performed. There is no stress concentration and little difference in stress distribution on both left and right sides in spite of the asymmetrical mandibular shape and masticatory condition. The numerical result suggests mechanical adaptation of mandible by the masticatory forces.

Keywords: muscular force, masticatory system, estimation method, finite element analysis, patient-specific model, objective function.
\end{abstract}

\section{Introduction}

Patient-specific simulation with FEM is a significant technique for examination of biomechanical characteristics of bones. There are three important procedures to obtain reliable results in the FEM analysis:

- Accurate modeling of the complex bony shape.

- Proper setting of the material property.

- Proper setting of the boundary conditions.

In order to perform the first procedure, we proposed patient-specific modeling method based on X-ray CT images [1]. For the second procedure, we assigned Young's moduli for each element of the model referring to the CT value [2]. 
This paper mainly focuses on the third procedure; "How to set the boundary conditions to the model." We have to estimate proper muscular forces to examine in vivo biomechanical characteristics of bones because we cannot directly measure muscular forces under a non-invasive condition. We briefly describe our patientspecific modeling method at the beginning, then propose a method to estimate masticatory forces.
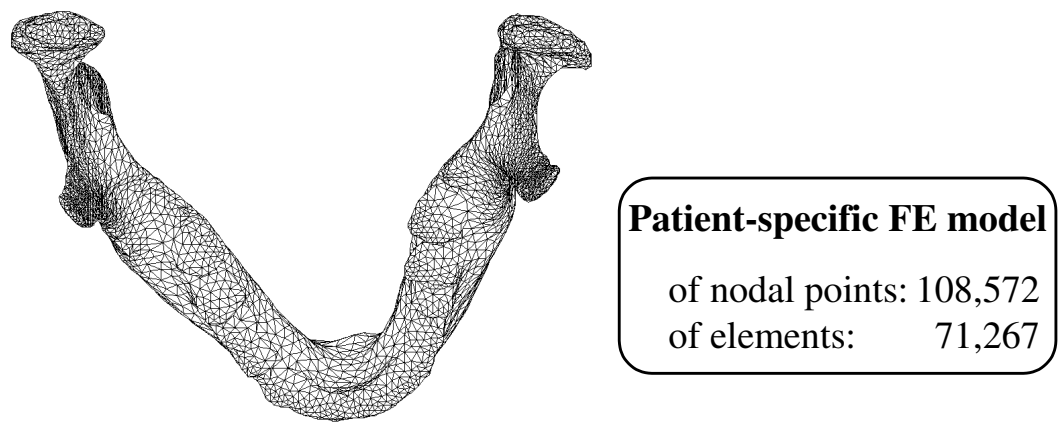

Top view

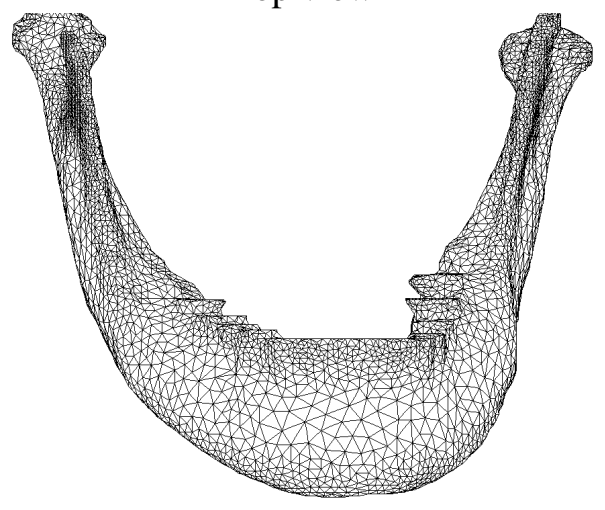

Front view

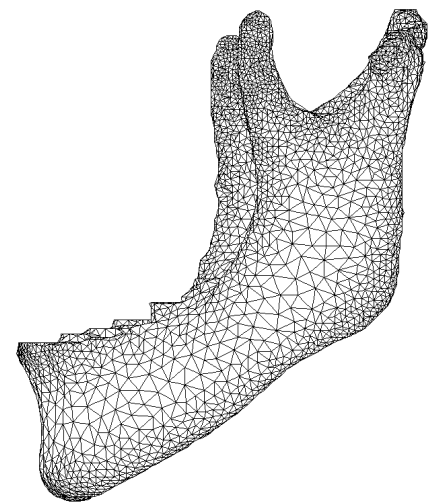

Right view

Figure 1: Patient-specific model of a human mandible based on the X-ray CT images.

\section{Materials and methods}

\subsection{Patient-specific FE modeling}

The patient-specific model is generated by our proposed modeling method [1]. The method automatically generates a finite element model with tetrahedral elements based on the X-ray CT images. We applied the method to a human mandible 
which has a severe deformity. Figure 1 shows a patient-specific model of a human mandible generated by the proposed method.

Material properties of the model are assigned to each element of the model based on the CT value [2]. That is, we assume that bone mineral density $\left[\mathrm{g} / \mathrm{cm}^{3}\right]$ is proportional to CT value. The relationship between the bone density $(\rho)$ and CT value $\left(V_{C T}\right)$ can be approximated by the relation

$$
\rho=a \cdot V_{C T}+b
$$

where $a$ and $b$ are constants associated with the bone density and CT value.

There are several reports about the relationship between the bone density and Young's modulus [3] [5]. This study refers to the report by Carter et al. [3] and calculates Young's moduli $(E)$ of each element using the equation

$$
E=c \cdot \dot{\varepsilon}^{0.06} \cdot \rho^{3}
$$

where $\dot{\varepsilon}$ is a strain rate and $c$ is a constant value. We assume $\dot{\varepsilon}$ as $1.0 \mathrm{sec}^{-1}$, and select the value of $c$ so that the highest $\mathrm{CT}$ value corresponds to $E=16 \mathrm{GPa}$.

\subsection{Estimation of masticatory forces}

Living bones are usually affected by multiple muscles. In order to execute stress analyses of any musculoskeletal system, we must determine definite values of the muscular forces. There are several reports on numerical methods to estimate muscular forces at human limbs $[6,7]$. The methods are based on efficiency of muscular activities. However, we should take notice that there is a great difference in terms of shape and function between a human mandible and a limb. This paper proposes a new objective function for estimation of the human mandible.

Estimation of masticatory forces using the objective function is performed by the following five steps:

Step 1 Loads and directions of biting forces and muscular forces are applied to the model.

Step 2 Moment around the axis between two condyles is balanced.

Step 3 Reaction forces at joints are computed.

Step 4 Activity rates of muscles are adjusted by an optimization method.

Step 5 Go to Step 2 until acquiring a convergent solution.

In step 1, attachment sites of the muscles and the muscular directions are set to the finite element model. In order to analyze stress distribution of the mandible, we consider five kinds of masticatory muscles as shown in Figure 2(a): masseter, anterior temporalis, posterior temporalis, medial pterygoid, and lateral pterygoid. These muscular forces are distributed to the corresponding nodal points of the finite element model as boundary condition. This procedure usually takes a lot of time because general purposed software tools called pre-post processor are not suit 


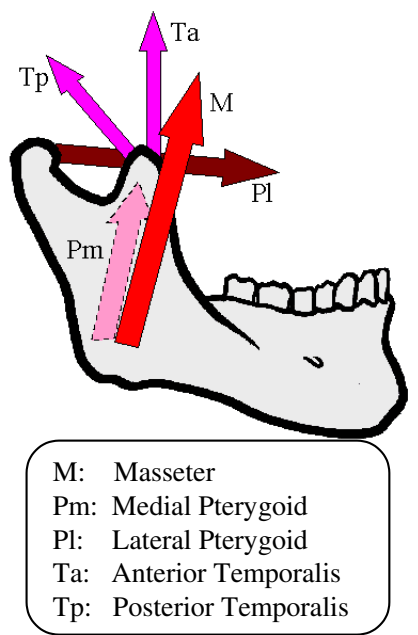

(a) Masticatory muscles for stress analysis

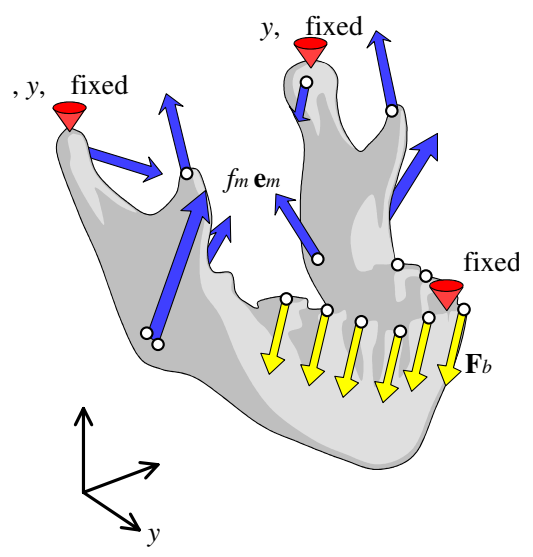

(b) Mechanical support condition

Figure 2: Mechanical conditions in occlusion.

for analyses of bones. So, we developed the setting support system of boundary conditions for easy operation [8].

Step 2 computes muscular forces under the moment balance of the model. There are three constraint points as shown in Figure 2(b). The moment balance around the $x$-axis between two condyles of the mandible can be adjusted by the function

$$
\sum_{i=1}^{b}\left(\mathbf{r}_{x i}^{B} \times \mathbf{F}_{i}^{B}\right)+\alpha \sum_{i=1}^{m}\left(\mathbf{r}_{x i}^{M} \times f_{i}^{M} \mathbf{e}_{i}^{M}\right)=0
$$

where $b$ and $m$ represent the number of biting teeth and muscles. $\mathbf{F}_{i}^{B}$ is a vector of $i$ th biting force. $\mathbf{r}_{x i}^{B}$ and $\mathbf{r}_{x i}^{M}$ are vectors from the $x$-axis to the loading points on the teeth and attachment sites of muscles. $f_{i}^{M}$ denotes the activity rate of $i$ th muscle, and $\mathbf{e}_{i}^{M}$ is an unit vector which has an direction of the muscle. This equation defines the definite value of muscular force $\alpha f_{m}$.

Step 3 computes the reaction forces $\left(\mathbf{F}_{1}^{R}\right.$ and $\mathbf{F}_{2}^{R}$ ) acting on the joint portions using the equation

$$
\mathbf{F}_{1}^{R}+\mathbf{F}_{2}^{R}+\sum_{i=1}^{b} \mathbf{F}_{i}^{B}+\sum_{i=1}^{m} \alpha f_{i}^{M} \mathbf{e}_{i}^{M}=0 .
$$

The forces are distributed around the constraint points of the finite element model to reduce stress concentration.

Step 4 successively adjusts activities of muscles using an optimization method. In this study, we focus on the mechanical characteristics of the masticatory system and provide the following three kinds of criteria considering the shape and function of the human mandible. 
- summation of square of muscular forces.

- moment around saggital axis (y-axis.)

- summation of square of reaction forces at condyles.

In the previous paper [8], we proposed an estimation method that considered first and second criteria. That is, we estimated the masticatory forces with the consideration of the efficiency of muscular activities and moment balance. In this paper, we introduce the third criterion that reaction force at the condyles should be small because contact area of a condyle is rather small. In consideration of the three criteria, we propose the optimization function of the form

$$
\begin{aligned}
I= & \sum_{i=1}^{m}\left(\alpha f_{i}^{M}\right)^{2} \\
& +k_{1}\left|\sum_{i=1}^{b}\left(\mathbf{r}_{y i}^{B} \times \mathbf{F}_{i}^{B}\right)+\sum_{i=1}^{m}\left(\mathbf{r}_{y i}^{M} \times f_{i}^{M} \mathbf{e}_{i}^{M}\right)\right| \\
& +k_{2}\left(\left|\mathbf{F}_{1}^{R}\right|^{2}+\left|\mathbf{F}_{2}^{R}\right|^{2}\right)
\end{aligned}
$$

where $k_{1}$ and $k_{2}$ are weighting coefficient combining the three criteria. $k_{1}$ is the coefficient of the moment around $y$-axis that coincides with the center of gravity of the biting forces. $k_{2}$ is the coefficient of the summation of square of reaction forces at condyles. $k_{1}$ is expected to be a large value because the moment around the $y$-axis should be converged to zero and sum of muscular forces represented by the first term is quite huge compared with the value of the moment. The third term, square of reaction forces at condyles is desirable to be small but will not be zero. Therefore $k_{2}$ is expected to be a modest value.

The minimization is solved by the steepest descent method under the condition that all muscular forces are positive and the masseter produces the maximum muscular force.

\subsection{Setting support system of boundary conditions}

We used the setting support system [8] to set up boundary condition of the finite element model. There are two important operations when we set up the boundary condition. First, we must specify attachment regions of muscles to the mandible. In the finite element analyses of musculoskeletal system, distributed forces are applied to nodal points corresponding to muscular sites. Second, we must set up proper directions of muscular forces and biting forces to the model according to the anatomical knowledge.

Our developed system realizes following functions with simple mouse operation; selection of nodal points on muscular attachment sites, dispersion of the muscular forces, setting of the direction of the muscular forces and calculation of the reaction forces around a joint portion. Figure 3 shows the boundary condition of the model set up with the our setting support system. 


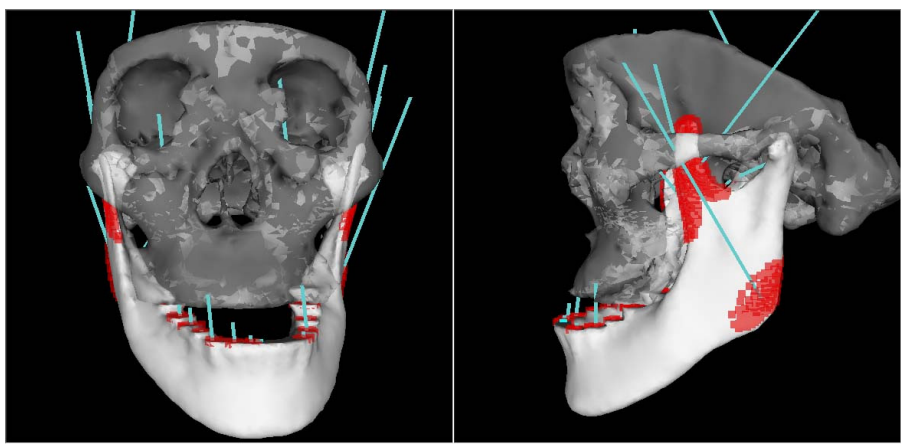

Figure 3: Setting of the boundary condition of the masticatory system.

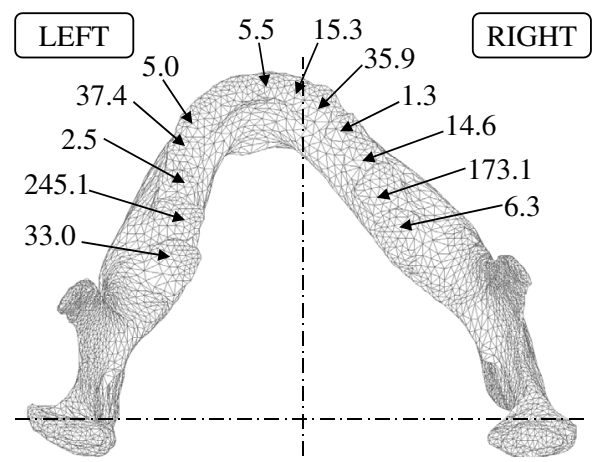

Figure 4: The distributions of biting forces [N].

\section{Results and discussion}

The proposed method was applied to a male patient aged 18 years whose jaw has a severe deformity. Figure 4 shows the reaction forces at the teeth determined by a pressure sheet. There were large differences in the biting forces between left-side and right-side. At the same time, surface electromyographies (EMGs) were measured from masseter (M), anterior temporalis (Ta) and posterior temporalis (Tp) of the patient. Since it is difficult to measure EMG of medial pterygoid (Pm) and lateral pterygoid $(\mathrm{Pl})$, their activities were estimated by our medical collaborative researcher.

In this study, we focused on the influence of $k_{1}$ and $k_{2}$ in the objective function. The estimations of the muscular forces and stress analyses were performed under following three cases;

(a) $k_{1}=0.0, k_{2}=0.0$

(b) $k_{1}=1000.0, k_{2}=0.0$

(c) $k_{1}=1000.0, k_{2}=10.0$

That is, the case (a) only considers efficiency of muscular activities. The case (b) 


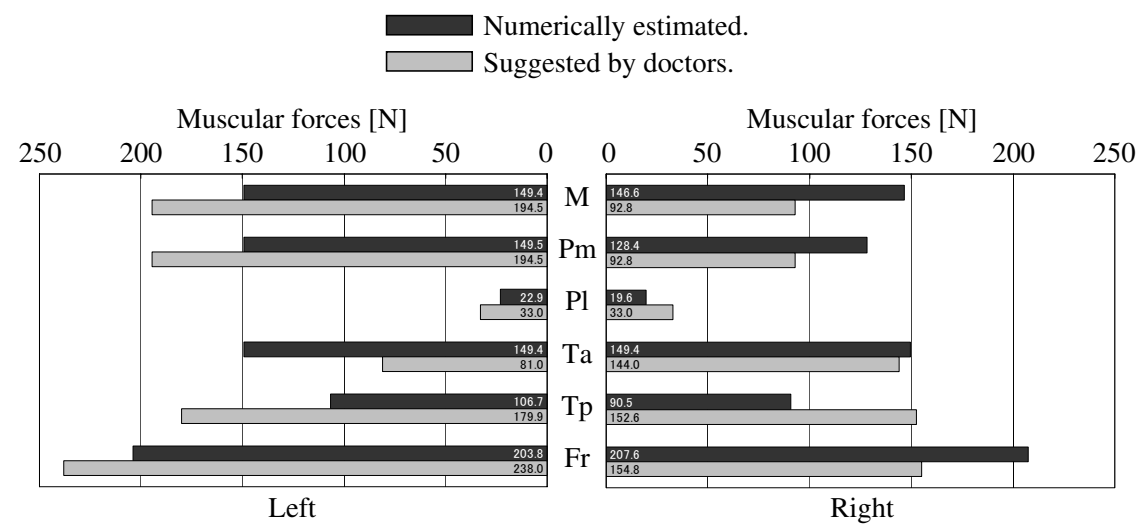

(a) $k_{1}=0.0, k_{2}=0.0$

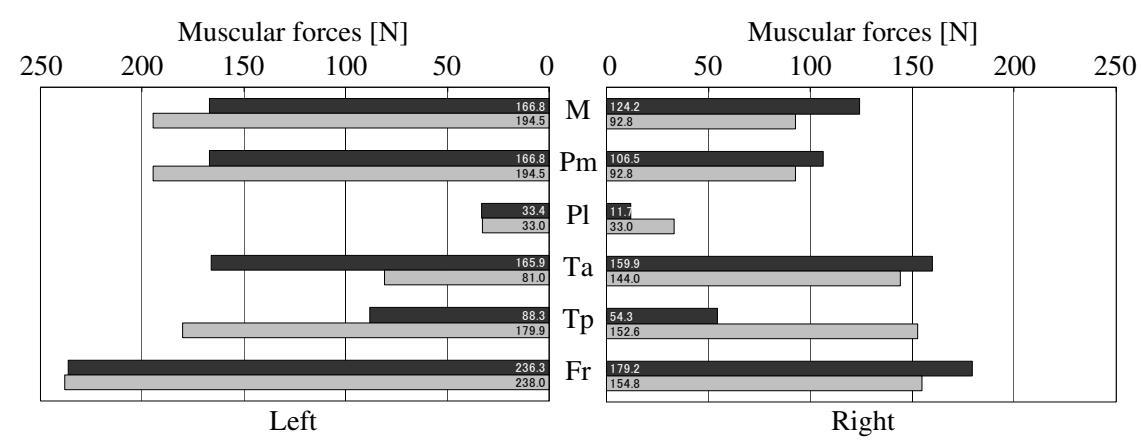

(b) $k_{1}=1000.0, k_{2}=0.0$

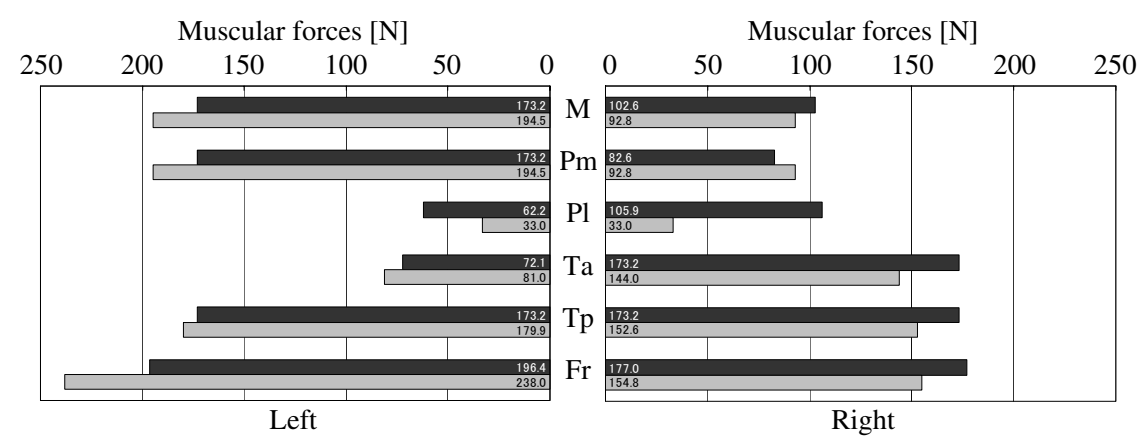

(c) $k_{1}=1000.0, k_{2}=10.0$

Figure 5: Estimated muscular forces and calculated reaction forces at condyles.

deals not only the efficiency but also the moment balance between muscular and biting forces. The case (c) integrates the three criteria including minimization of reaction forces at condyles. 


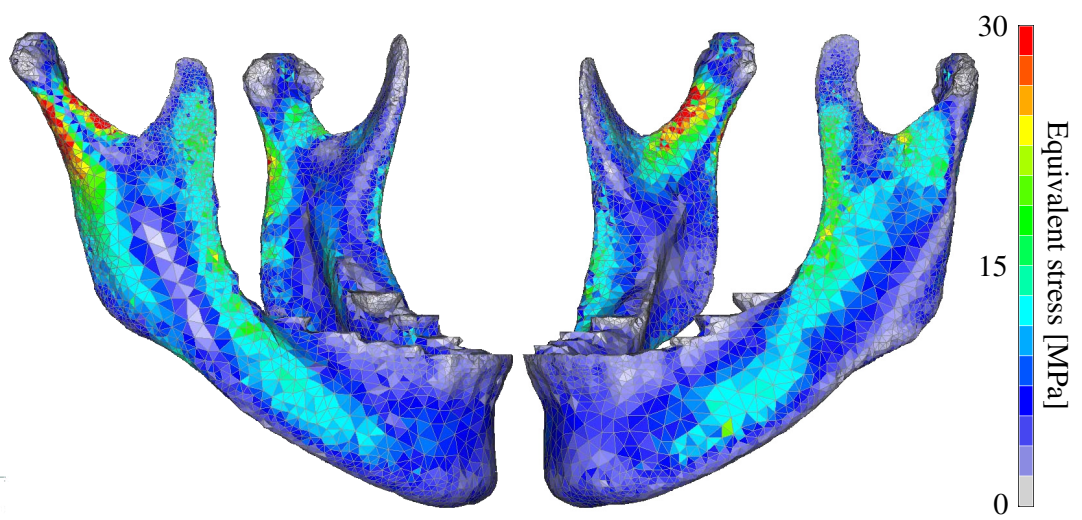

(a) $k_{1}=0.0, k_{2}=0.0$

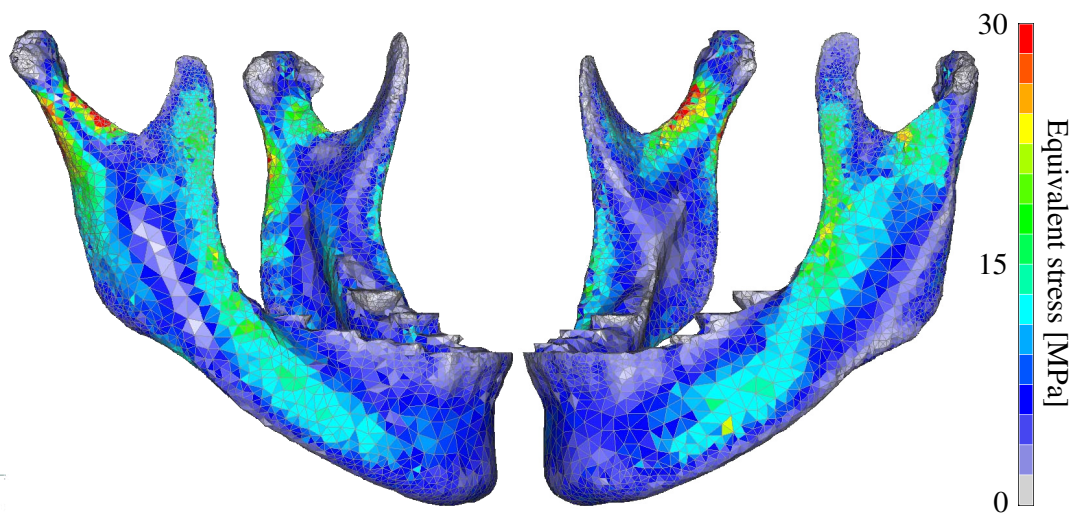

(b) $k_{1}=1000.0, k_{2}=0.0$
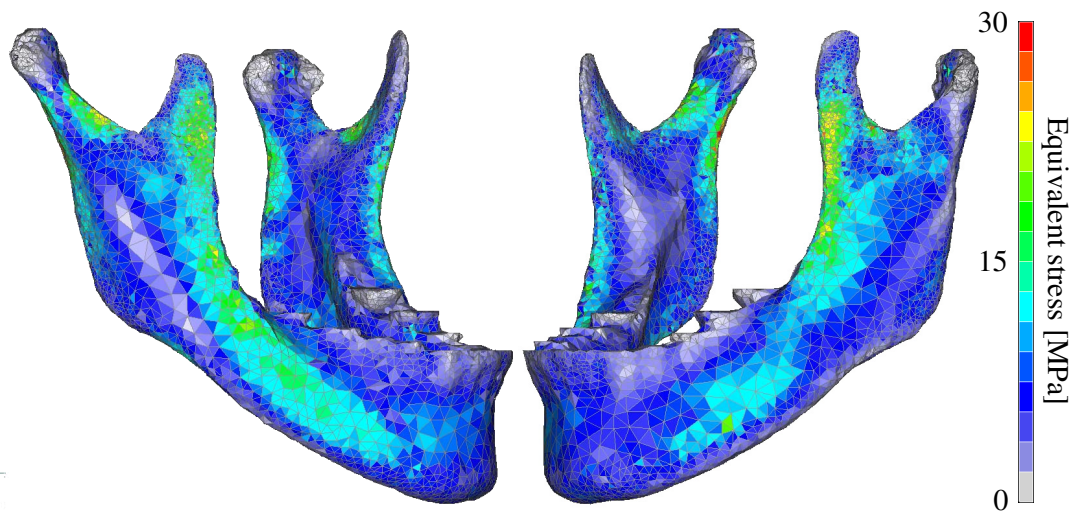

(c) $k_{1}=1000.0, k_{2}=10.0$

Figure 6: Stress distribution under the estimated mechanical conditions. 


\subsection{Estimated muscular forces}

Figure 5 summarizes the estimated muscular forces in the each case. Light gray bars in the figure indicate muscular forces suggested by the medical researcher based on the analysis of EMG signals. Dark gray bars present numerically estimated forces based on the proposed method.

Figure 5(a) shows the estimated muscular forces in the case: $k_{1}=0.0, k_{2}=0.0$. In spite of the quite asymmetrical mandibular shape, the estimated result implies symmetrical muscular forces and equal reaction forces at condyles. This condition seems to be improbable.

Figure 5(b) presents the estimation in the case: $k_{1}=1000.0, k_{2}=0.0$. Owing to the addition of the moment balance, the muscular forces of the numerical result clearly become asymmetric on each side. This suggests availability of the addition of the moment balance. However, estimated forces of anterior temporalis are larger than posterior ones on both sides, even though EMG signals show posterior ones work harder.

Figure 5(c) shows the estimated result using the proposed method in this paper; in the case: $k_{1}=1000.0, k_{2}=10.0$. The muscular forces of the numerical result well agree to the suggestion by the medical researcher based on the analysis of EMG, except for the forces by lateral pterygoid (Pl.) The suggested forces of lateral pterygoid is rather tentative because it is very difficult to measure the EMG signal of lateral pterygoid in the current stage of technology. It is said that the lateral pterygoid works to open the mouth, but there is a possibility that the muscle works to reduce the reaction forces at condyles in the state of maximum occlusion.

\subsection{Computed stress distributions}

Figure 6 (a) $\sim(c)$ show the computational results of equivalent stress distributions under each estimated muscular forces. The analytical results in the cases of (a) and (b), there are high stress concentrations at the right condyler neck portion. On the contrary, There are lower stresses in the whole mandible and the distribution shows little difference on both sides in the case of (c). The uniformed stress distribution suggests mechanical adaptation of the human mandible.

We also performed stress analysis under the condition based on the analysis of EMG signals. The stress distribution based on the EMG was similar to that based on the estimated muscular forces in the case (c). This shows the validity of the proposed method.

\section{Conclusion}

In this study, we examined the estimation method of muscular forces on the viewpoint of shape and function of masticatory system. Muscular forces were estimated using the objective function composed of three criteria. The computational results revealed that the objective function was valid to estimate accurate masticatory forces. 
This study treated only one patient. We would like to apply the proposed method to many patients and examine the effectivity of the method in our further study.

\section{References}

[1] Inou, N., Koseki, M., Jonishi, M. and Maki, K., Automated Individual Modeling Method Based on the Multi-sliced Images, Proc. IASTED Intr. Conf. BIOMECH., pp.142-145, 2004.

[2] Koseki, M., Kitagawa, Y., Inou, N. and Maki, K. A Correction Method of CT Values Influenced by Partial Volume Effect, Proc. First Asian Pacific Conf. Biomech., (04-204), pp.119-120, 2004.

[3] Carter, D. R. and Hayes, W. C., The Compressive Behavior of Bone as a Two-Phase Porous Structure, J. Bone Joint Surg., 59-A, pp.954-962, 1977.

[4] Keyak, J. H., Lee, I. Y. and Skinner, H. B., Correlations between orthogonal mechanical properties and density of trabecular bone: Use of different densitometric measures, J. Biomed. Mat. Res., 28, pp.1329-1336, 1994.

[5] Keller, T. S., Predicting the Compressive Mechanical Behavior of Bone, $J$. Biomech., 27-9, pp.1159-1168, 1994.

[6] Crowninshield, R. D., Use of Optimization Techniques to Predict Muscle Forces, Trans. ASME, 100, pp.88-92, 1978.

[7] Yoshinari, S., Tadano, S. and Ukai, T., Muscle Force Analysis during Three-Dimensional Motion of the Forearm, Proc. Intr. Conf. New Frontiers Biomech. Eng., pp.317-320, 1997.

[8] Inou, N., Koseki, M., Tanizaki, H. and Maki, K., Development of the Support System for Individual Stress Analyses of a Bone, Proc 6th Intr. Symp. Comp. Meth. Biomech. Biomed. Eng., 109A(CD-ROM), 2004. 